Supporting Information

\title{
Toward Inexpensive Photocatalytic Hydrogen Evolution: A Nickel Sulfide Catalyst Supported on a High-Stability Metal-Organic Framework
}

\author{
Aaron W. Peters, ${ }^{1}$ Zhanyong Li, ${ }^{1}$ Omar K. Farha, ${ }^{1,2^{*}}$ and Joseph T. Hupp ${ }^{1^{*}}$ \\ ${ }^{1}$ Department of Chemistry, Northwestern University, 2145 Sheridan Road, Evanston, Illinois 60208, \\ United States \\ ${ }^{2}$ Department of Chemistry, Faculty of Science, King Abdulaziz University, Jeddah 21589, Saudi Arabia \\ *Email: o-farha@northwestern.edu, j-hupp@northwestern.edu
}




\section{Table of Contents}

$\begin{array}{ll}\text { Physical methods and instrumentation } & \mathrm{S}-4\end{array}$

Photocatalytic $\mathrm{H}_{2}$ Evolution $\quad \mathrm{S}-4$

Quantum Yield Calculation $\quad$ S-5

Figure S1: Photograph image of NU-1000, NiS-AIM, and Ni-AIM S-6

Figure S2: PXRD patterns of NiS-AIM (blue), NU-1000 (red), and a simulated S-6 pattern from crystallographic data (black).

Figure S3 - (a) $\mathrm{N}_{2}$ adsorption-desorption isotherms of the parent material, NU- $\quad \mathrm{S}-7$ 1000, and the NiS metallated NU-1000 (NiS-AIM). Calculated BET surface areas correspond to $2200 \mathrm{~m}^{2} / \mathrm{g}$ and $1210 \mathrm{~m}^{2} / \mathrm{g}$ for NU-100O and NiS-AIM, respectively. (b) DFT pore-size distributions calculated from $\mathrm{N}_{2}$ isotherms showing a slight decrease in the mesopore from 29 to $27 \AA$ after nickel sulfide metalation.

Figure S4. SEM-EDS line scans of NiS-AIM material. (a) SEM image of NiSAIM before catalysis (b) corresponding line scan through the crystal showing uniform distribution of nickel and sulfur (c) SEM image of NiS-AIM after catalysis (d) corresponding line scan of NiS-AIM after photocatalytic hydrogen production catalysis.

Figure S5: DRIFTS spectra of NU-1000 and NiS-AIM. (a) Full spectra. (b) Region associated with the terminal $-\mathrm{OH}$ and $\mu_{3}-\mathrm{OH}$ stretching region on the node of NU-1000. (c) Region associated with hydrogen bound aqua groups on the node.

Figure S6: Typical gas chromatograms from a series of times taken throughout photocatalytic experiment. (a) the full spectrum. Asterisks $\left(^{*}\right)$ at $1.55,1.95$, and 3.50 min denote a valve switch, $\mathrm{CO}_{2}$, and $\mathrm{O}_{2}$, respectively. $\mathrm{CO}_{2}$ and $\mathrm{O}_{2}$ are impurities from the syringe. (b) A zoomed in portion of the chromatogram showing the $\mathrm{H}_{2}$ peak at $3.24 \mathrm{~min}$.

Figure S7: Structure of the dye used in this study, rose-bengal. Figure S8: Beer's Law plot of a solution of rose-bengal in $\mathrm{pH} 7$ tris(hydroxylmethyl)aminomethane buffer.

Figure S9: Photocatalytic hydrogen evolution with visible light of a bulk, $\quad$ S-10 commercially available, NiS catalyst with the presence of the rose-bengal dye. Figure S10: Comparison of diffuse-reflectance UV-Vis spectra of assynthesized NiS-AIM and after photocatalytic hydrogen evolution in which rosebengal has been included in the solution and allowed to adsorb through the MOF

Figure S11: Photocatalytic irradiation of NiS-AIM and rose-bengal with visible light over the course of $10 \mathrm{~h}$ showing degradation of the dye after prolonged exposure to light.

Figure S12: Photocatalytic hydrogen evolution after NiS-AIM was filtered and new dye was added to the filtrate. Rates of formation of $\mathrm{H} 2$ are significantly decreased compared to NiS-AIM

Table S1: A summary of the turnover numbers calculated for the photocatalytic reactions in this study 
Figure S13: Photocatalytic $\mathrm{H}_{2}$ evolution rates with different sacrificial

reagents. Triethanolamine is the only sacrificial reagent tested that gave decent yields.

Figure S14: A comparison of XPS spectra before (a-c) and after $(d-f)$ photocatalytic hydrogen evolution showing the $\mathrm{Zr} 3 \mathrm{~d}$ region $(\mathrm{a}+\mathrm{d})$, $\mathrm{Ni} 2 \mathrm{p}$ region $(b+e)$ and $S 2 p$ region $(c+f)$.

Figure S15: (a) PXRD patterns of simulated NU-1000 (black), NU-100o (red), as-synthesized NiS-AIM (blue), and NiS-AIM after photocatalytic hydrogen evolution (green). (b) $\mathrm{N}_{2}$ isotherms of NU-1000 (red squares), NiS-AIM (blue diamonds), and NiS-AIM after catalysis (green triangles) and (c) their corresponding pore size distributions. 


\section{Physical Methods and Instrumentation}

$\mathrm{N}_{2}$ isotherms were collected on a Micromeretics Tristar II 3020 instrument at $77 \mathrm{~K}$. Pore-size distributions were calculated from these isotherms using a carbon split-pore model with a $\mathrm{N}_{2}$ kernal.

Raman spectra were collected on a Horiba LabRAM HR Confocal Raman system. A small amount of the sample was placed on a piece of carbon tape and the sample was irradiated with a $785 \mathrm{~nm}$ laser through a 50\% ND filter for $120 \mathrm{~s}$ and average over three scans.

Diffuse reflectance infrared Fourier transform spectra (DRIFTS) were recorded on a Nicolet 7600 FT-IR spectrometer equipped with an MCT detector. Samples diluted in KBr were measured with a $\mathrm{KBr}$ background. The spectra were collected at $1 \mathrm{~cm}^{-1}$ resolution and $128 \mathrm{scans}$

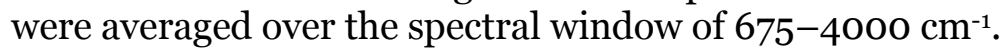

Diffuse reflectance UV-vis spectra were measured on a Perkin Elmer LAMBDA 1050 spectrophotometer with an $150 \mathrm{~mm}$ integrating sphere accessory. Solid samples were placed on a PXRD plate and covered with a quartz slide. Measurements were made within the $700-300$ nm spectral window.

Fluorescence specta were collected on a Jovin Yvon Spex FluoroLog-3 fluorimeter.

Electronic absorption UV-vis spectra were collected on a Cary 5000 (Varian) spectrometer.

Gas chromatography was performed on a Agilent 7890 A GC system equipped with a TCD detector and an HP-Molesieve and HP-Plot Q columns with $\mathrm{N}_{2}$ as a carrier gas.

Atomic layer deposition was performed in a Savannah S100 (Cambridge Nanotech, Inc) in a custom-made stainless steel powder sample holder.

Powder X-ray diffraction patterns were collected on a ATX-G (Rigaku) instrument equipped with a $18 \mathrm{~kW}$ copper rotating anode $\mathrm{x}$-ray source. Roughly $10 \mathrm{mg}$ of sample was loaded onto a powder sample holder and mounted on the instrument. Samples were recorded from $1.5<2 \theta<20^{\circ}$ at a scan rate of $2^{\circ} / \mathrm{min}$ and a step size of $0.05^{\circ}$.

X-ray photoelectron spectra (XPS) were collected using a Thermo Scientific ESCALAB 250 Xi (Al Ka radiation, $h v=1486.6 \mathrm{eV}$ ). Samples were irradiated with an electron flood gun and all spectra were referenced to the $\mathrm{C}$ is peak $(284.8 \mathrm{eV})$.

Inductively coupled plasma-atomic emission spectroscopy data were collected on Varian VistaMDX model ICP-AES spectrometer. Generally, the MOF sample was digested in $1.0 \mathrm{~mL}$ nitric acid and $0.25 \mathrm{~mL} 30 \% \mathrm{H}_{2} \mathrm{O}_{2}$ followed by heating in a Biotage SPX microwave reactor at $150{ }^{\circ} \mathrm{C}$ for $5 \mathrm{~min}$. This solution was then diluted with MilliQ water to a final volume of $15 \mathrm{~mL}$.

Scanning electron microscopy images were collected on a Hitachi SU8030 FE-SEM microscope with a field emission gun. Samples were coated with $\mathrm{OsO}_{4}$ at $\sim 7 \mathrm{~nm}$ of thickness with a Denton Desk III TSC Sputter Coater.

\section{Photocatalytic Hydrogen Evolution}

The catalyst ( $5 \mathrm{mg}, 2 . \mathrm{O} \times 10^{-3} \mathrm{mmol}$ ), Rose Bengal $\left(1.5 \mathrm{mg}, 1.5 \times 10^{-3} \mathrm{mmol}\right)$, triethanolamine (o.089 g, o.60 mmol)1.0 M pH 7 tris(hyrdoxymethyl)aminomethane, were added to a 5-mL biotage microwave vial charged with a stir bar. The vial was capped with a septum and degassed 
with $\mathrm{N}_{2}$ gas for 20 minutes. The vial was then irradiated with light filtered with an AM-1.5 solar filter from a FluoroLog-3 fluorimeter with slits adjusted so that a power density of $100 \mathrm{~mW} / \mathrm{cm}^{2}$ irradiated on the solution. Approximately every 30 mins, $200 \mu \mathrm{L}$ of the gas mixture in the head space was drawn injected into the GC to quantify the amount of $\mathrm{H}_{2}$ being formed.

Gas Chromatography Protocol

The gas phase products were analyzed and quantified by injection a $200 \mu \mathrm{L}$ sample of the headspace of the reaction flask into the GC. A GC valve (G: On/Off) technique was used.

Column 1: HP-Plot Q

Column 2: HP-Plot Molesieve

Detector: Thermal Conductivity Detector

Carrier Gas: $\mathrm{N}_{2}$ at $8 \mathrm{~mL} / \mathrm{s}$

Oven Temperature: $40{ }^{\circ} \mathrm{C}$

Event Sequence: G-On at o min, G-Off at $1.55 \mathrm{~min}$

Under these conditions, the retentions times of $\mathrm{CO}_{2}, \mathrm{H}_{2}$, and $\mathrm{O}_{2}$ are 1.95, 3.24, and 3.50 min, respectively. Peaks attributed to $\mathrm{CO}_{2}$ and $\mathrm{O}_{2}$ are from the syringe. The peak attributed to $\mathrm{H}_{2}$ was integrated and referenced against a standard calibration curve of various injections from a $2 \%$ $\mathrm{H}_{2}$ bal. $\mathrm{N}_{2}$ gas cylinder.

\section{Quantum Yield Calculation}

Apparent quantum yield was calculated from the equations below where $\eta_{\mathrm{H} 2}$ is the number of moles of $\mathrm{H}_{2}$ produced, $N_{A}$ is Avogadro's number, $h$ is planks constant, $c$ is the speed of light, $P$ is the power density of the light source, $S$ is the irradiation area, $\lambda$ is the wavelength of the irradiation light, and $t$ is the amount of time the sample was irradiated with light.

$$
\begin{gathered}
\text { A.Q.Y }=\frac{\# \text { reacted } e^{-}}{\# \text { of absorbed photons }}=\frac{2 \times \mathrm{H}_{2} \text { produced }}{\# \text { of absorbed photons }} \\
\# \text { incident photons }=\frac{E_{\text {tot }}}{E_{\text {photon }}}=\frac{P S \lambda t}{h c} \\
\text { A.Q.Y }(\%)=\frac{2 \eta_{\mathrm{H}_{2}} N_{A} h c}{P S \lambda t} \times 100
\end{gathered}
$$




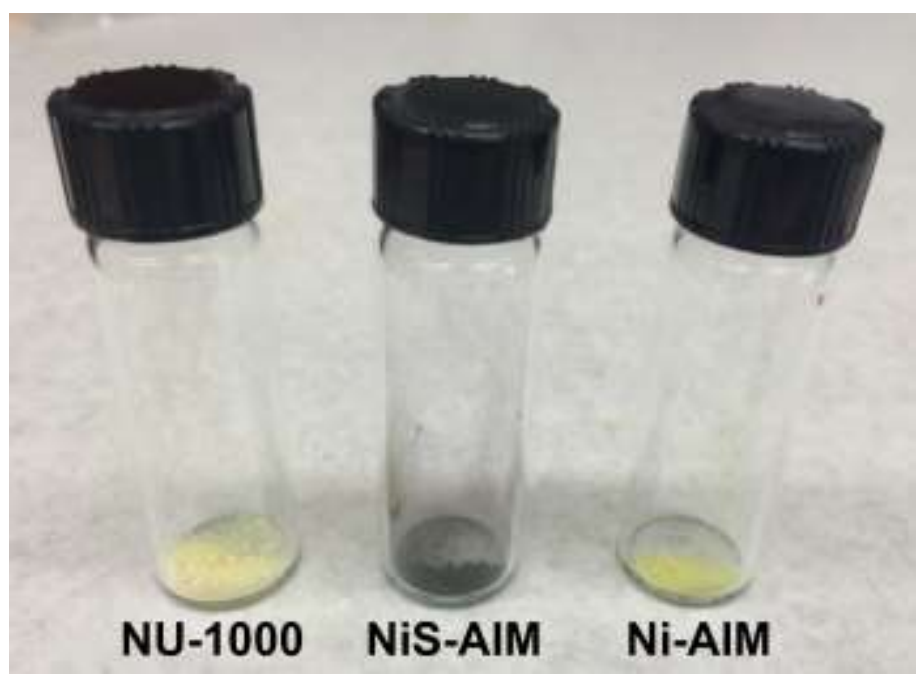

Figure S1: Photograph image of NU-1000, NiS-AIM, and Ni-AIM

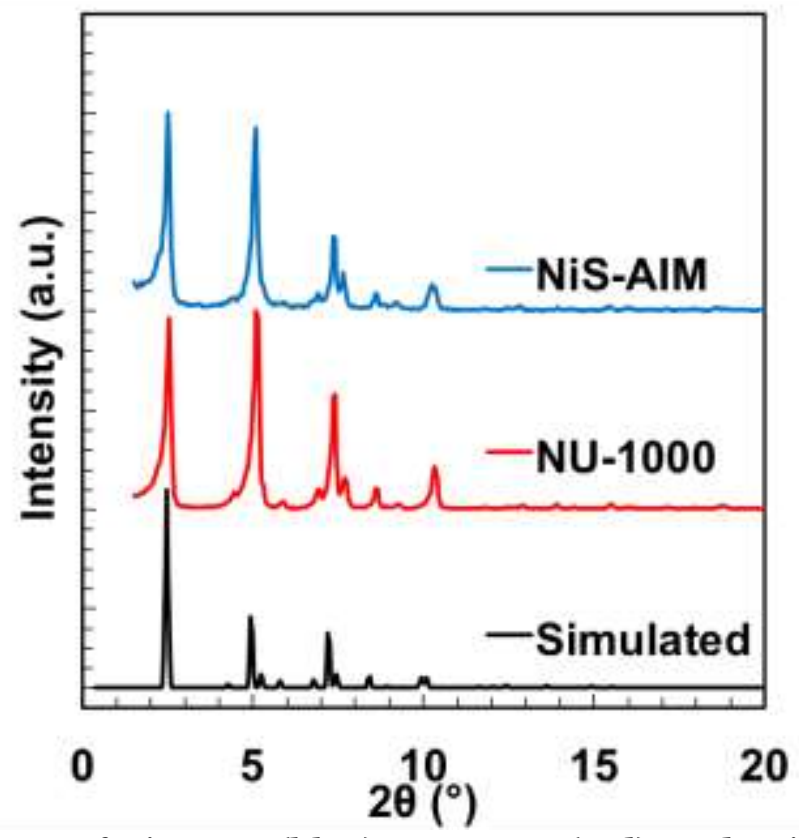

Figure S2: PXRD patterns of NiS-AIM (blue), NU-1000 (red), and a simulated pattern from crystallographic data (black). No assignable peaks for crystalline morphologies of nickel sulfide (e.g. $\mathrm{NiS}, \mathrm{Ni}_{3} \mathrm{~S}_{4}, \mathrm{NiS}_{2}$ etc.) are observed here or at higher angles due to one AB cycle of ALD producing an amorphous $\mathrm{NiS}_{\mathrm{x}}$ species coordinated to the node. 

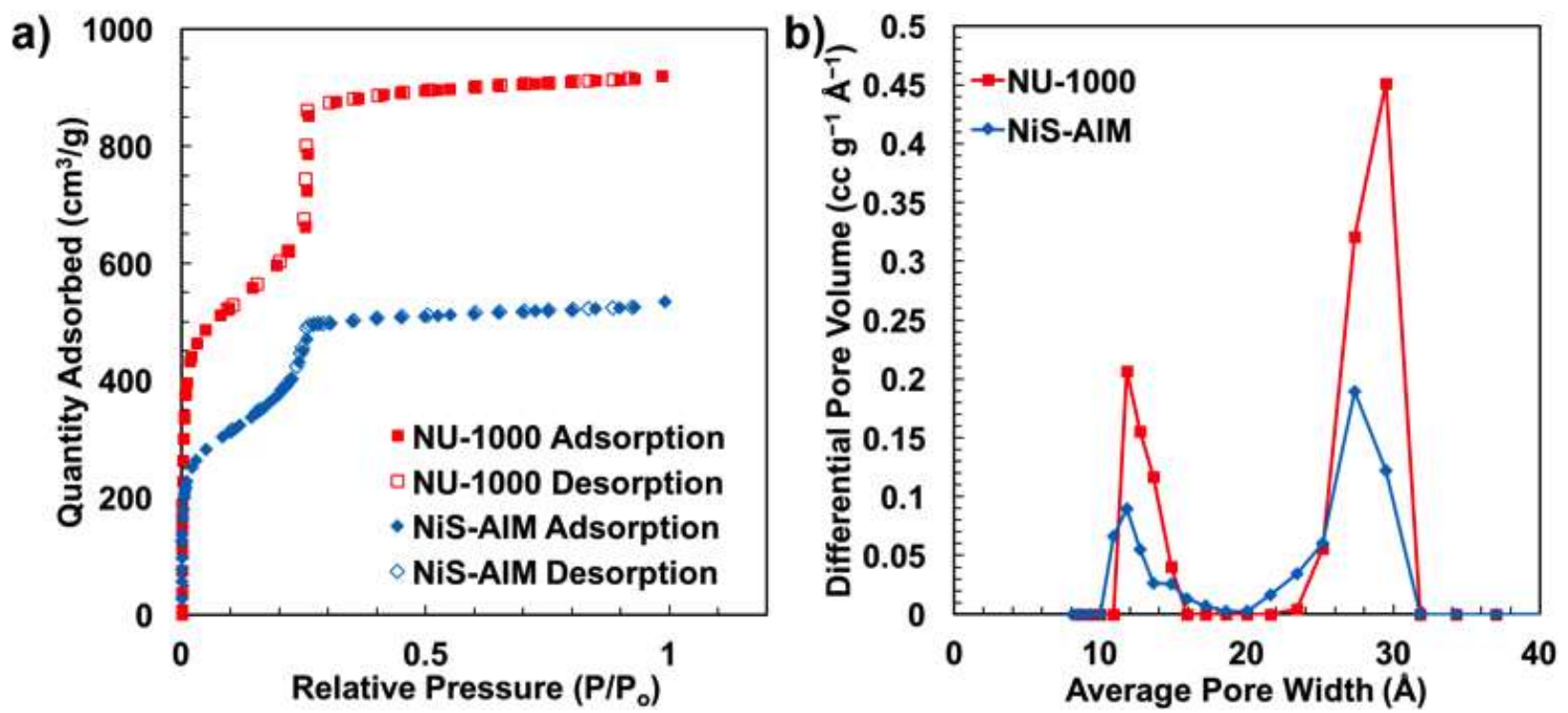

Figure S3. (a) $\mathrm{N}_{2}$ adsorption-desorption isotherms of the parent material, NU-1000, and the NiS metallated NU-1000 (NiS-AIM). Calculated BET surface areas correspond to $2200 \mathrm{~m}^{2} / \mathrm{g}$ and $1210 \mathrm{~m}^{2} / \mathrm{g}$ for NU-100O and NiS-AIM, respectively. (b) DFT pore-size distributions calculated from $\mathrm{N}_{2}$ isotherms showing a slight decrease in the mesopore from 29 to $27 \AA$ after nickel sulfide metalation.
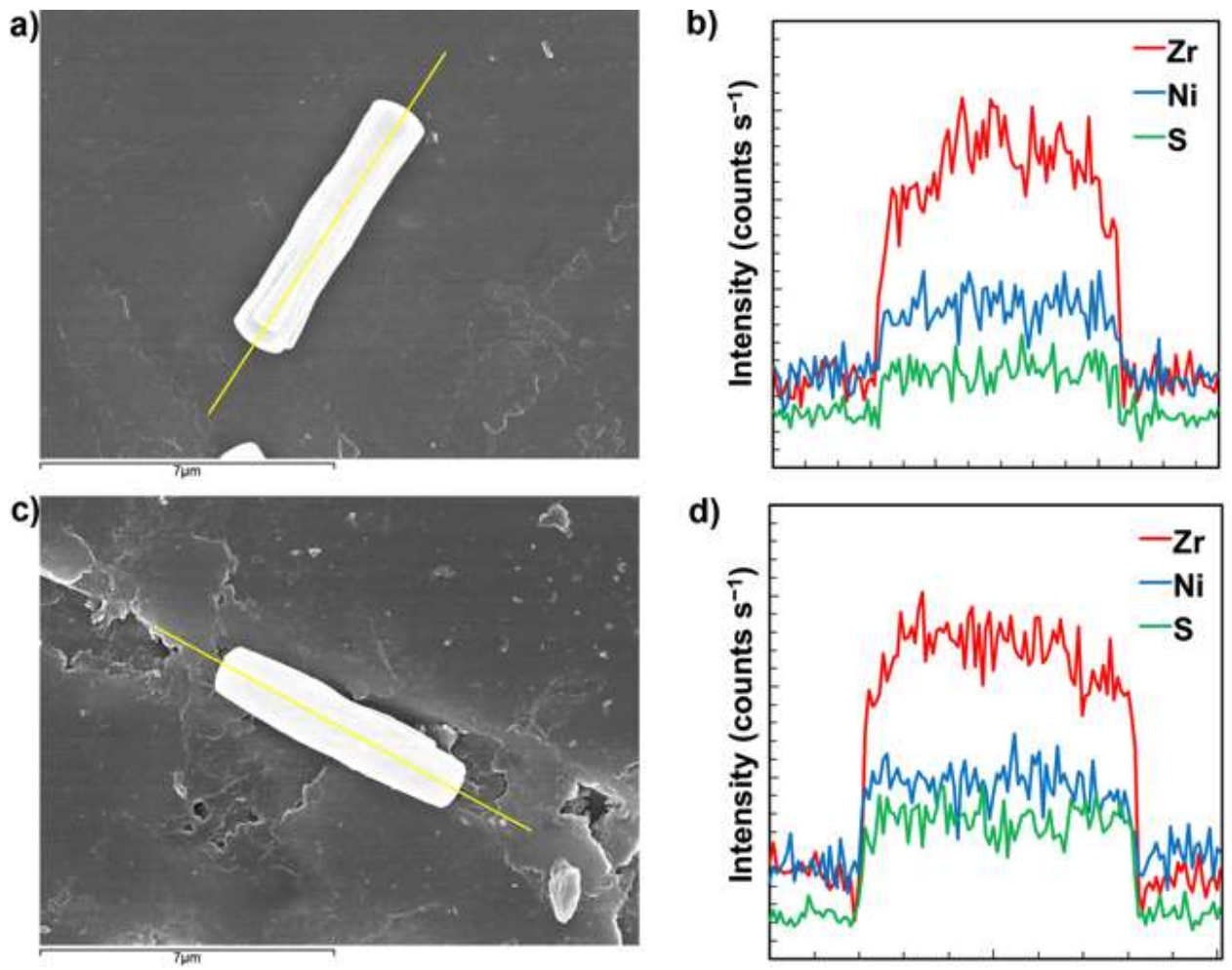

Figure S4. SEM-EDS line scans of NiS-AIM material. (a) SEM image of NiS-AIM before catalysis (b) corresponding line scan through the crystal showing uniform distribution of nickel and sulfur (c) SEM image of NiS-AIM after catalysis (d) corresponding line scan of NiS-AIM after photocatalytic hydrogen production catalysis. 
a)

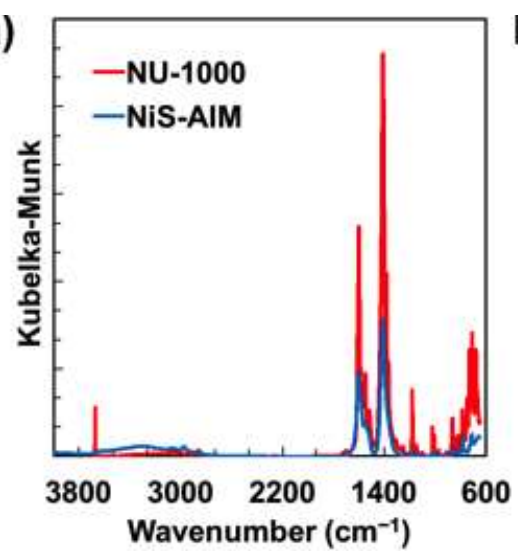

b)

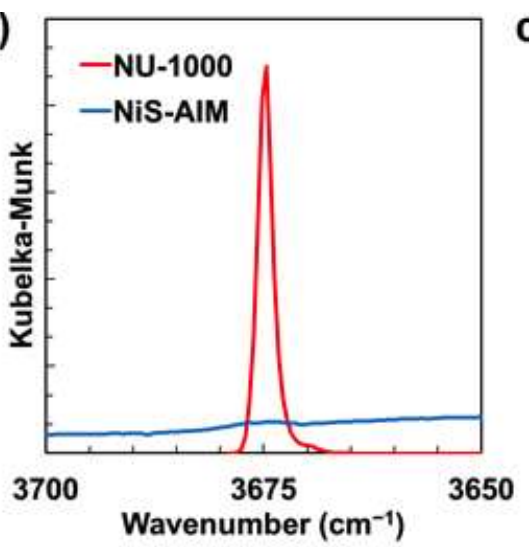

c)

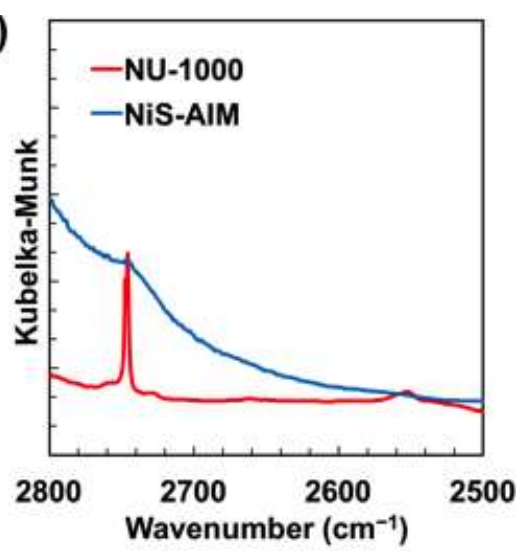

Figure S5: DRIFTS spectra of NU-100o and NiS-AIM. (a) Full spectra. (b) Region associated with the terminal $-\mathrm{OH}$ and $\mu_{3}-\mathrm{OH}$ stretching region on the node of $\mathrm{NU}-1000$. (c) Region associated with hydrogen bound aqua groups on the node.

a)

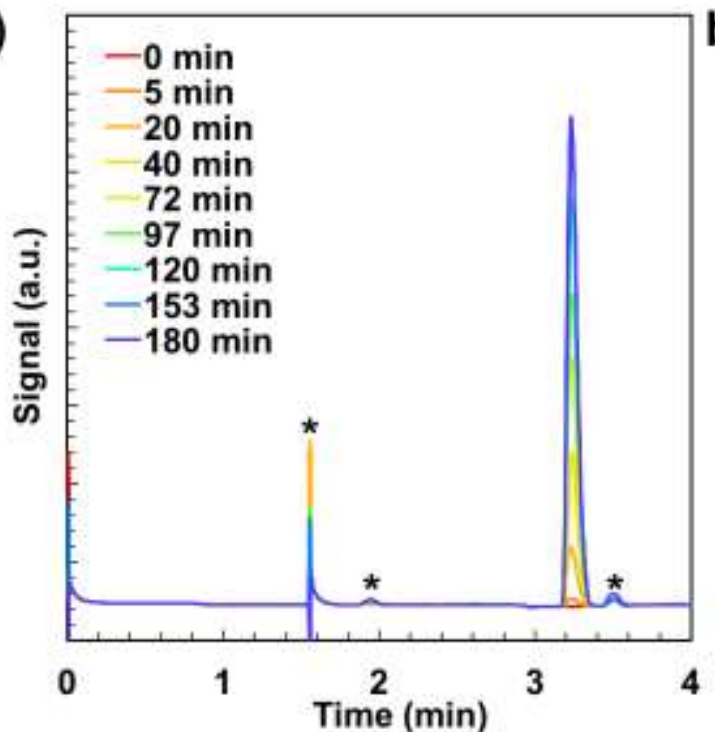

b)

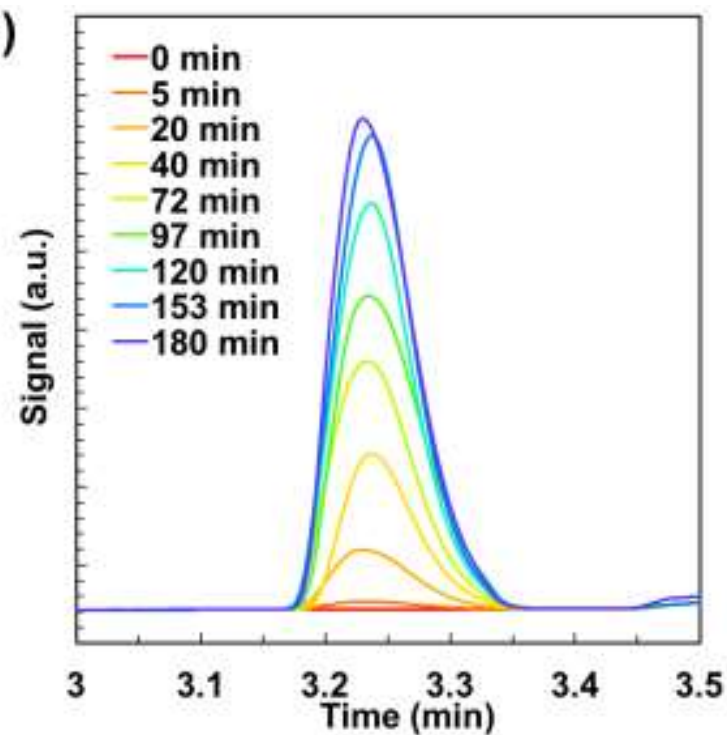

Figure S6: Typical gas chromatograms from a series of times taken throughout photocatalytic experiment. (a) the full spectrum. Asterisks $(*)$ at 1.55, 1.95, and 3.50 min denote a valve switch, $\mathrm{CO}_{2}$, and $\mathrm{O}_{2}$, respectively. $\mathrm{CO}_{2}$ and $\mathrm{O}_{2}$ are impurities from the syringe. (b) A zoomed in portion of the chromatogram showing the $\mathrm{H}_{2}$ peak at $3.24 \mathrm{~min}$. 


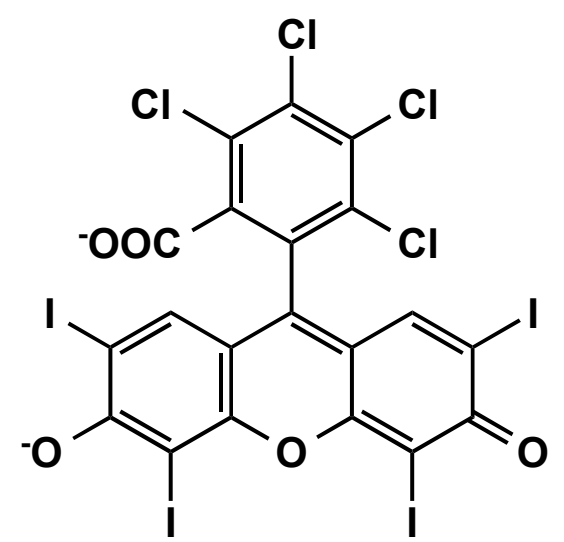

Figure S7: Structure of the dye used in this study, rose-bengal.

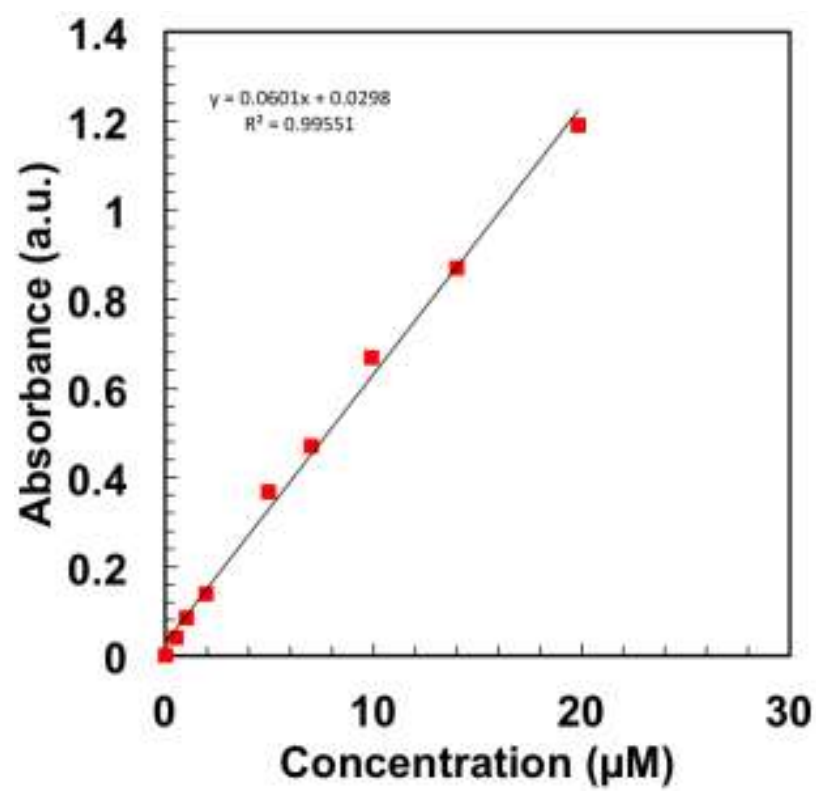

Figure S8: Beer's Law plot of a solution of rose-bengal in $\mathrm{pH} 7$ tris(hydroxylmethyl)aminomethane buffer. 


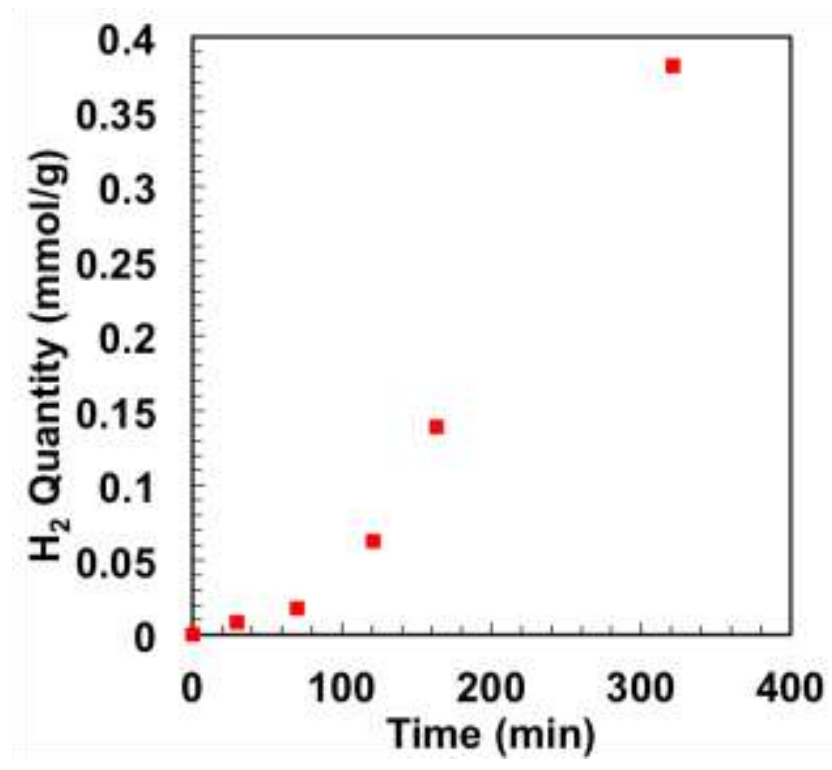

Figure S9: Photocatalytic hydrogen evolution with visible light of a bulk, commercially available, NiS catalyst in the presence of the rose-bengal dye.

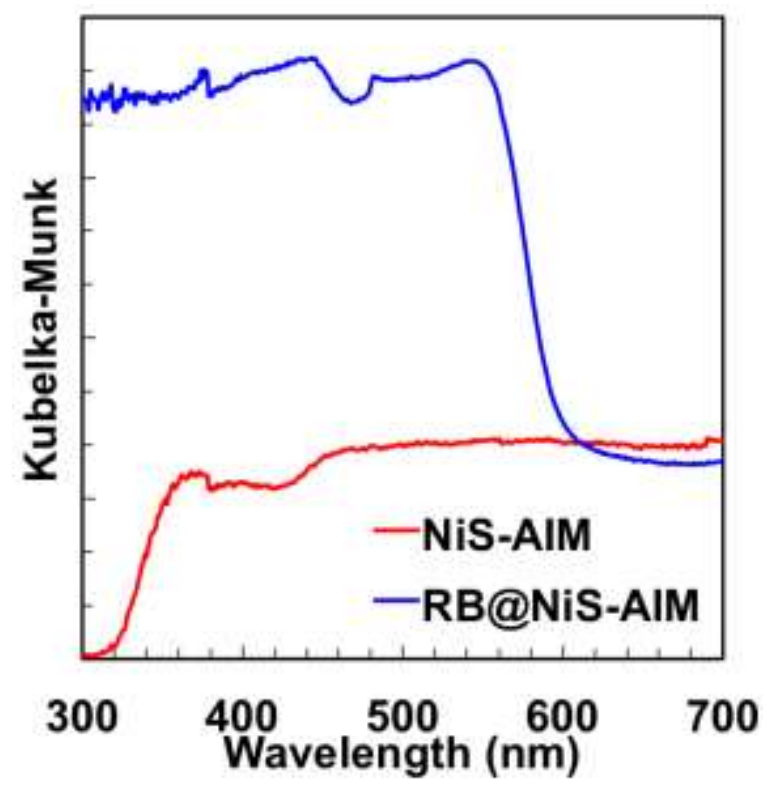

Figure S10: Comparison of diffuse-reflectance UV-Vis spectra of as-synthesized NiS-AIM and after photocatalytic hydrogen evolution in which rose-bengal has been included in the solution and allowed to adsorb through the MOF 


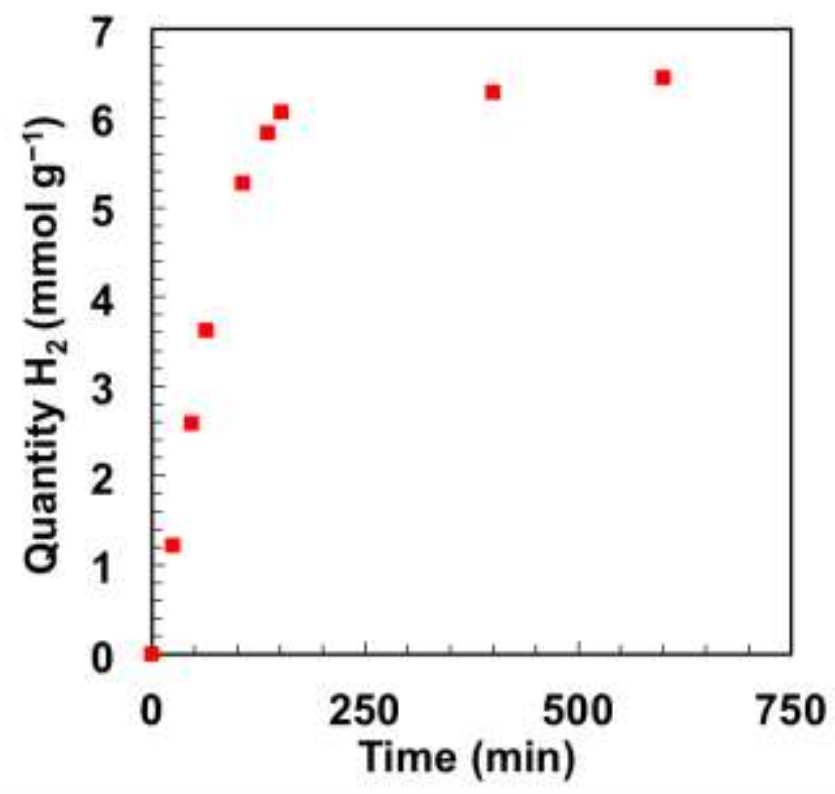

Figure S11: Photocatalytic irradiation of NiS-AIM and rose-bengal with visible light over the course of $10 \mathrm{~h}$ showing degradation of the dye after prolonged exposure to light.

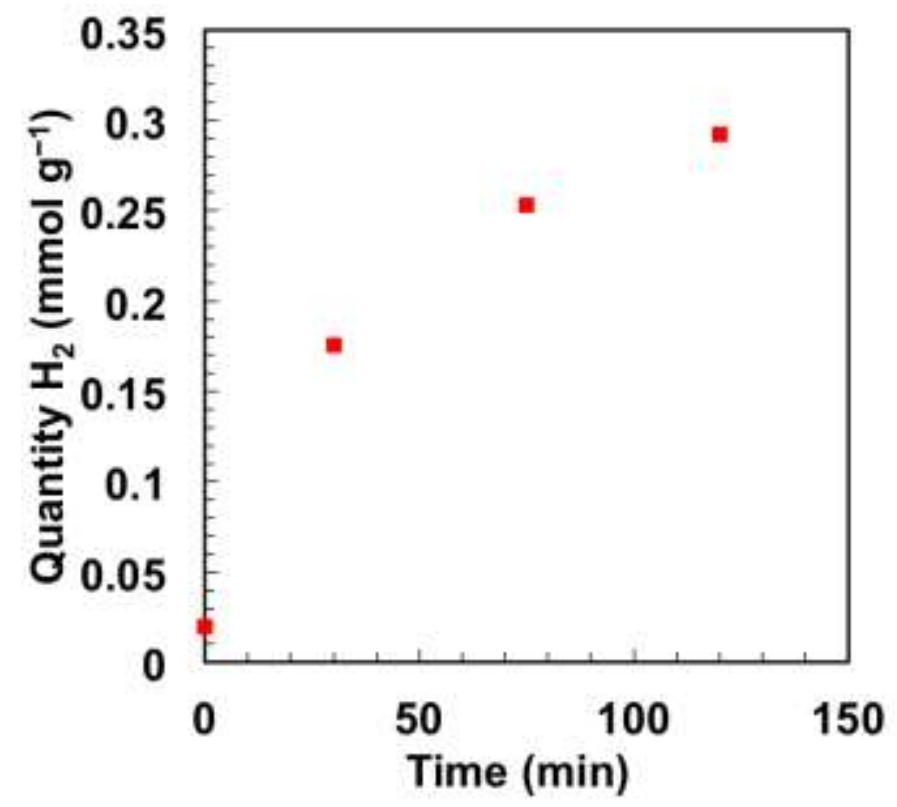

Figure S12: Photocatalytic hydrogen evolution after NiS-AIM was filtered and new dye was added to the filtrate. Rates of formation of $\mathrm{H} 2$ are significantly decreased compared to NiS-AIM 
Table S1: A summary of the turnover numbers calculated for the photocatalytic reactions in this study

\begin{tabular}{|c|c|}
\hline Catalyst & TONa $\left(\times 10^{-2}\right)$ \\
\hline NiS-AIM $^{b}$ & 220 \\
\hline NU-1000 ${ }^{b}$ & N.A. \\
\hline NiS-AIMc & 0.31 \\
\hline NiS-AIMc,d & 600 \\
\hline NiS-AIM (1st Recycle)c,d & 550 \\
\hline NiS-AIM (2nd Recycle)c,d & 480 \\
\hline NiS Bulk ${ }^{\mathrm{c}, \mathrm{d}}$ & 0.56 \\
\hline $\mathrm{NiO}-\mathrm{AIM} \mathrm{c}, \mathrm{d}$ & 0.91 \\
\hline ZnS-AIMc,d & N.A. \\
\hline
\end{tabular}

aTON $=\left(\right.$ mole $\mathrm{H}_{2}$ produced/total mole of $\mathrm{Ni}_{\mathrm{x}} \mathrm{S}_{\mathrm{y}}$ in reaction flask) bIrradiated with UV light. cIrradiated with visible light through AM-1.5 global filter, $100 \mathrm{~mW} / \mathrm{cm}^{2}$. ${ }^{\mathrm{d}}$ Rose-bengal $\left(1.5 \times 10^{-}\right.$ $3 \mathrm{mmol}$ ) added to solution.

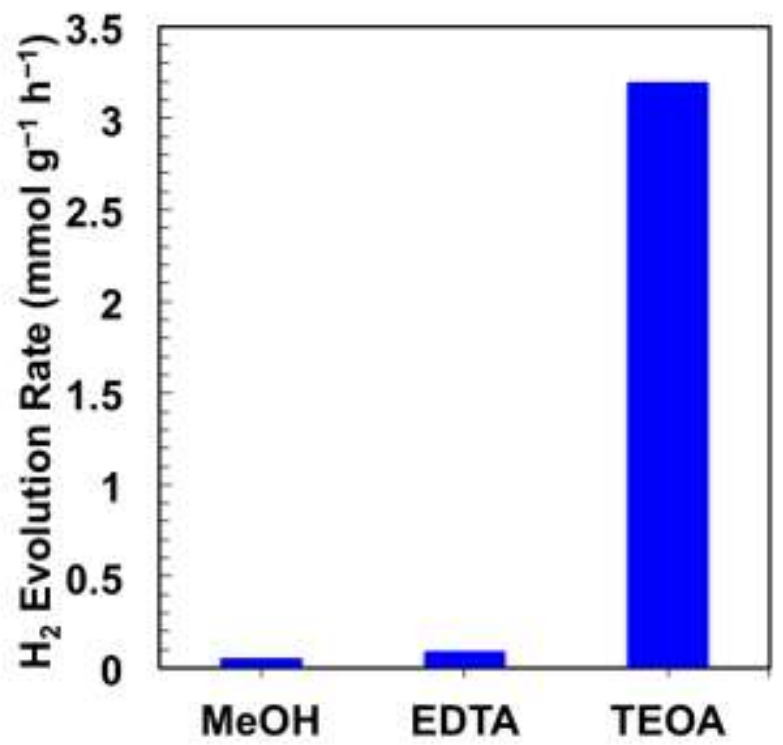

Figure S13: Photocatalytic $\mathrm{H}_{2}$ evolution rates with different sacrificial reagents. Triethanolamine is the only sacrificial reagent tested that gave decent yields. 

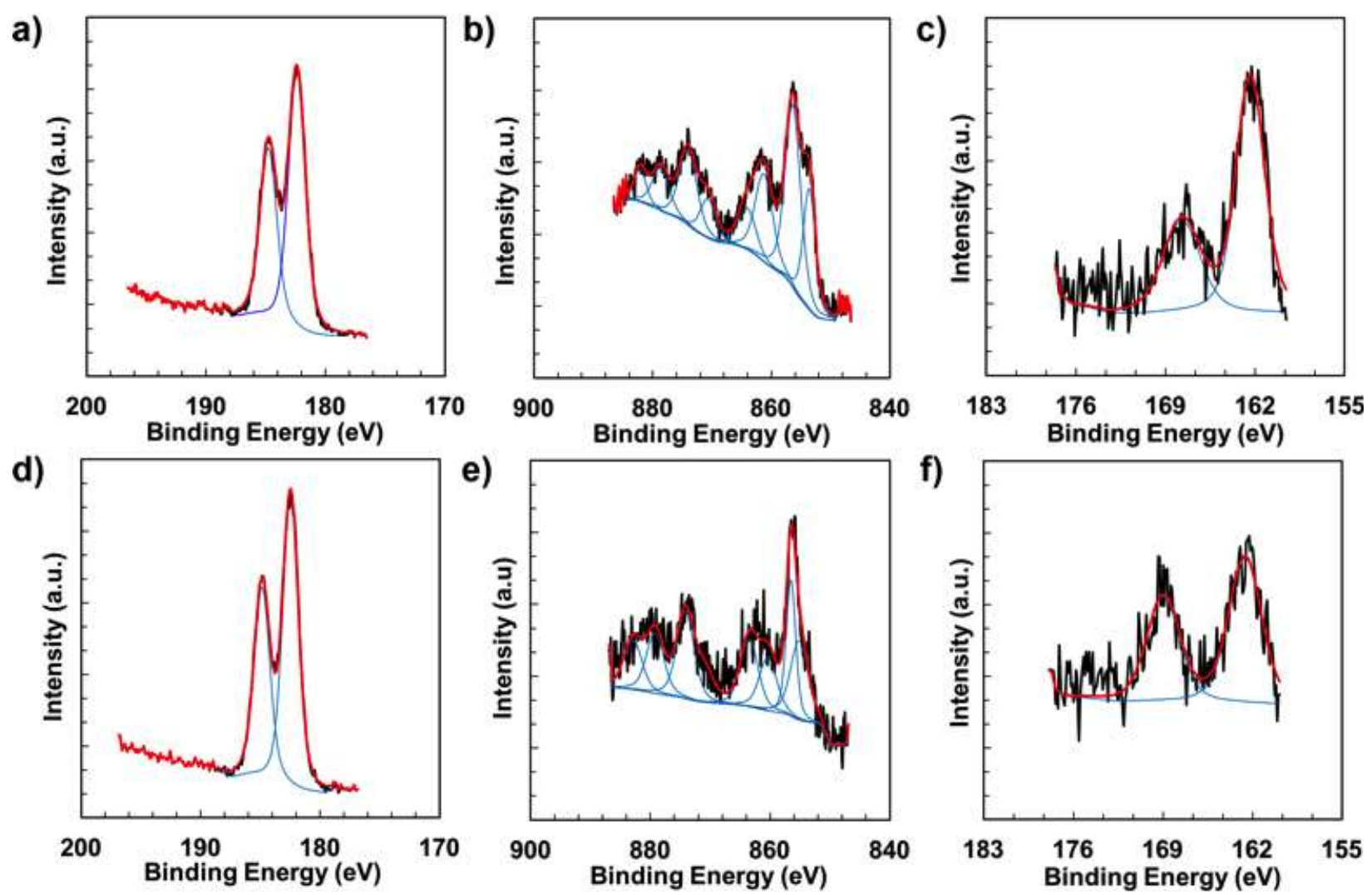

Figure S14: A comparison of XPS spectra before $(a-c)$ and after $(d-f)$ photocatalytic hydrogen evolution showing the $\mathrm{Zr} 3 \mathrm{~d}$ region $(\mathrm{a}+\mathrm{d})$, $\mathrm{Ni} 2 \mathrm{p}$ region $(\mathrm{b}+\mathrm{e})$ and $\mathrm{S} 2 \mathrm{p}$ region $(\mathrm{c}+\mathrm{f})$.
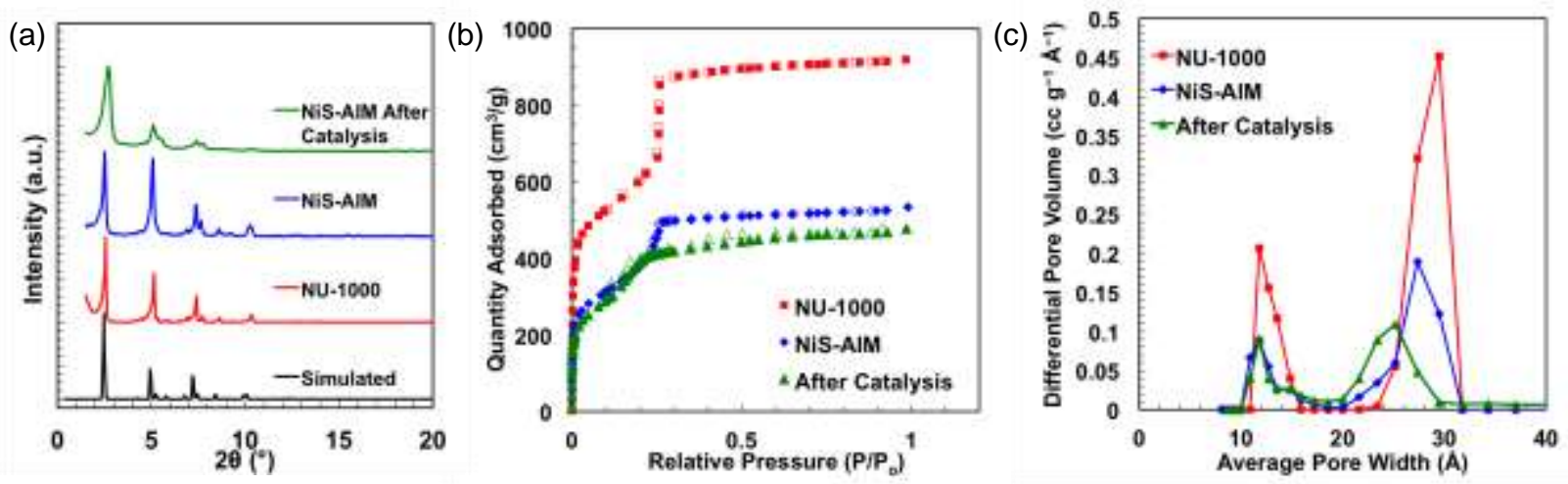

Figure S15: (a) PXRD patterns of simulated NU-1000 (black), NU-100o (red), as-synthesized NiS-AIM (blue), and NiS-AIM after photocatalytic hydrogen evolution (green). (b) $\mathrm{N}_{2}$ isotherms of NU-1000 (red squares), NiS-AIM (blue diamonds), and NiS-AIM after catalysis (green triangles) and (c) their corresponding pore size distributions. 